\title{
Pathologie de l'articulation temporo-mandibulaire: apport de l'IRM dans l'appréciation des désunions condylo-discales ou luxations discales
}

\author{
Léon $\mathbf{H}^{1}$, Teissier $\mathrm{JM}^{2}$ \\ ${ }^{1}$ Service d'Odontologie et de Prothèse maxillo-faciale, CHU, Nîmes, France \\ ${ }^{2}$ Service d'Imagerie médicales, Hôpital privé des Franciscaines, Nîmes, France \\ herve.leon@chu-nimes.fr
}

Les pathologies de l'articulation temporo-mandibulaire sont toujours au centre de discussions passionnées. L'étiopathogénie et ses conséquences ne sont toujours pas clairement établies. Il est donc indispensable d'apporter le plus d'informations possible sur l'état articulaire dans le diagnostic, élément initial et obligatoire avant toute thérapeutique.

L'imagerie par résonance magnétique (IRM) est l'imagerie par excellence pour l'appréciation des désunions condylo-discales (luxations discales) mises en évidence lors de notre examen clinique. Aujourd'hui, nous les regroupons en deux types, les désunions condylo-discales réductibles (luxations discales réductibles) et les désunions condylo-discales irréductibles (luxations discales non réductibles). Grâce à la performance toujours plus poussée de cette technique d'imagerie et à l'expérience du radiologue et de l'odontologiste, il est possible de différencier plusieurs formes de désunions condylo-discales réductibles: celles par translation antéro-médiane et celles par rotation axiale. Cette précision paraît intéressante pour appréhender autrement les différentes désunions condylo-discales dans leur diagnostic et leur traitement. 